\title{
Co-localisation of Batrachochytrium dendrobatidis and keratin for enhanced diagnosis of chytridiomycosis in frogs
}

\author{
Veronica Olsen ${ }^{1}$, Alex D. Hyatt ${ }^{1, *}$, Donna G. Boyle ${ }^{1}$, Diana Mendez ${ }^{2}$ \\ ${ }^{1}$ CSIRO, Division of Livestock Industries, Australian Animal Health Laboratory, 5 Portarlington Road, East Geelong, \\ Victoria 3220, Australia \\ ${ }^{2}$ Amphibian Diseases Group, School of Public Health and Tropical Medicine, James Cook University, \\ Townsville, Queensland 4811, Australia
}

\begin{abstract}
Chytridiomycosis is a disease of post-metamorphic frogs caused by the fungus Batrachochytrium dendrobatidis and is associated with large declines in frog populations on a global scale. B. dendrobatidis is found only in the keratinised tissues, which include the mouthparts of healthy tadpoles. The epidermis of infected post-metamorphic frogs is thickened (hyperkeratosis) and the superficial layer can sometimes slough. Diagnosis is most commonly performed on stained sections of toe clips or ventral skin. Accurate interpretation can be difficult and requires a high level of expertise, particularly in infected animals exhibiting hyperkeratosis with sloughing. Misdiagnosis can occur when zoosporangia of $B$. dendrobatidis are shed with the superficial keratin layers. We have developed a staining protocol based on previously described methods to detect both $B$. dendrobatidis and keratin, to improve the sensitivity and specificity of diagnosis of chytridiomycosis by inexperienced diagnosticians.
\end{abstract}

KEY WORDS: Batrachochytrium dendrobatidis - Chytridiomycosis - Alkaline phosphatase · Hollande's Trichrome · Diagnostic assay

Resale or republication not permitted without written consent of the publisher

\section{INTRODUCTION}

Chytridiomycosis is a disease of post-metamorphic frogs caused by the fungus Batrachochytrium dendrobatidis, Class Chytridiomycetes. This class has >100 genera, including microscopic, zoospore-producing fungi that typically possess a single posterior flagellum; they are widespread in soil and water (Sparrow 1960, Fellers et al. 2001, Morehouse et al. 2003). Most are decomposers of cellulose, chitin, keratin, other fungi, algae and other plant material (Sparrow 1960, Powell 1993, Longcore et al. 1999). Berger et al. (1998), after studying moribund and dead adult frogs collected between 1993 and 1998 from sites of mass deaths in Australia and Panama, first described chytridiomycosis as a fungal disease affecting amphibians. Amphibians infected with $B$. dendrobatidis have since been reported from Africa, South, Central and North America, Europe, Australia and Oceania (R. Speare, available at www.jcu.edu.au/ school/phtm/PHTM/frogs/chyglob.htm).

Batrachochytrium dendrobatidis is only found in the keratinised tissues of amphibians, suggesting that it uses the keratin as a nutrient (Daszak et al. 1999). $B$. dendrobatidis can be carried by apparently healthy tadpoles in the keratinised mouthparts, often showing only abnormal mouthpart structures which lack pigmentation (Berger et al. 1999, Fellers et al. 2001, Nichols et al. 2001). In post-metamorphic frogs where clinical signs are too non-specific to be diagnostic, chytridiomycosis is diagnosed by laboratory tests. The epidermis adjacent to zoosporangia of $B$. dendrobatidis usually shows hyperkeratosis, not obvious clinically, but detectable upon microscopic examination. Postmetamorphic frogs can show more obvious clinical symptoms, including abnormal posture, lethargy and loss of righting reflex (R. Speare, available at www.jcu.edu.au/school/phtm/PHTM/frogs/chyglob.ht $\mathrm{m})$. In severely infected animals, lesions consist of abnormal epidermal sloughing, epidermal ulceration (including haemorrhages in the skin, muscle or eye), hyperaemia of digital and ventral skin, and congestion of viscera (Berger et al. 2000). The majority of diagnoses are made by gross observation of animals when 
symptoms become obvious, usually towards the final stages of infection.

Batrachochytrium dendrobatidis can be detected by routine histology using haematoxylin and eosin (HE) staining of skin sections and via direct examination of unstained smears of skin (Berger et al. 1999). A disadvantage of these techniques is that a high degree of expertise is required for accurate diagnosis (Longcore et al. 1999). More recently Berger et al. (2002) have described an immunoperoxidase (IPX) method using antibodies to specifically detect $B$. dendrobatidis. This protocol increases specificity and sensitivity of identification of $B$. dendrobatidis and decreases the need for expertise in pathology to make the diagnosis.

Diagnosis is straightforward in most cases of severe infection, as there are usually many zoosporangia of Batrachochytrium dendrobatidis (Berger et al. 1999). However, in some cases, positive diagnosis can be difficult even for the experienced diagnostician, particularly when the hyperkeratotic superficial epidermal layer sloughs. In such circumstances extensive sloughing of the skin may result in incorrect diagnosis or false negatives due to the absence of a highly infected surface layer (R. Speare, available at www.jcu.edu.au/ school/phtm/PHTM/frogs/chyglob.htm). In our laboratory we have noted (unpubl. results), as have Nichols et al. (2001), that shed pieces of skin from infected animals are associated with low to high numbers of $B$. dendrobatidis. To facilitate identification of $B$. dendrobatidis, a diagnostician should have a protocol where the presence of keratin is highlighted in addition to the zoosporangia, since, if the keratinised layer is missing from the epidermis, B. dendrobatidis will not be found.

This study describes a new combined procedure based on Berger et al.'s (2002) immunohistochemistry (IHC) method and Hollande's Trichrome keratin stain to simultaneously detect Batrachochytrium dendrobatidis and keratin.

\section{MATERIALS AND METHODS}

All tissues received for chytridiomycosis diagnosis were fixed in $10 \%$ neutral buffered formalin, processed through graded ethanols to xylene, and then embedded in paraffin. Sections were cut $4 \mu \mathrm{m}$ thick, collected onto washed glass slides and left to dry overnight at $37^{\circ} \mathrm{C}$.

Immunohistochemistry (IHC). Sections from infected frogs (e.g. Mixophes fasciolatus) were processed according to a modified version of the protocol described by Berger et al. (2002). In Berger et al. (2002), sections were incubated with immunoperoxidase (IPX) conjugated secondary antibody, resulting in red-brown staining of Batrachochytrium dendrobatidis set amongst the epithelial cells, counterstained with
Lillie-Mayer's haematoxylin (Sigma). Although it is not more effective as a substrate, alkaline phosphatase (AP) was preferred because of a bleaching effect on the IPX substrate by subsequent keratin staining, and the advantage of enhanced contrast between the substrate and keratin stains.

In the adapted AP protocol, sections were dewaxed with xylene and hydrated through graded ethanols to running tap water, then placed in distilled water. Sections were incubated with Rabbit 667 anti-chytrid polyclonal antibody (Berger et al. 2002), diluted to 1:1000 in $1 \%(\mathrm{w} / \mathrm{v})$ skim milk (Diploma)/tris buffered saline (TBS) for $45 \mathrm{~min}$ at $37^{\circ} \mathrm{C}$, washed for $5 \mathrm{~min}$ with TBS, and incubated with ENVISION anti-rabbit/ mouse AP (DakoCytomation) for $20 \mathrm{~min}$ at $37^{\circ} \mathrm{C}$. After washing again for 5 min with TBS, BCIP/NBT substrate (DakoCytomation) was added directly to the sections and incubated for $10 \mathrm{~min}$. Slides were well rinsed with distilled water, completing the procedure by which the chytrids were immuno-labelled. At this stage, if no further staining was required, slides were mounted in Geltol water-based gel (Immunon ${ }^{\mathrm{TM}}$, Thermo Shandon) and sealed with a coverslip.

The protocol described here also differs from that of Berger et al. (2002) in that the procedure takes approx. $2 \mathrm{~h}$ due to the substitution of ENVISION antirabbit/mouse AP and the elimination of the trypsin digestion step, compared to approx. $4 \mathrm{~h}$ for the LSAB 2-step kit (DakoCytomation).

Modified Hollande's Trichrome (keratin stain). To stain keratin, a modified staining protocol of Hollande's Trichrome keratin stain (Gray 1954) was performed. Slides were taken out of distilled water after immuno-labelling and incubated with $1 \%(\mathrm{w} / \mathrm{v})$ phosphomolybdic acid (Ajax/Univar) (Solution C) for 5 min at room temperature (RT), rinsed with distilled water, then incubated with saturated (minimum of $11 \% \mathrm{w} / \mathrm{v}$ ) Orange G (Gurr, Michrome \#411) solution (Solution D) for 5 min at RT. The majority of Solution D was decanted and $0.2 \%(\mathrm{w} / \mathrm{v})$ light green SF (Sigma; Solution E) was added without rinsing for 2 min at RT. Slides were subsequently placed without rinsing into $100 \%$ ethanol (2), cleared in xylene (2) and mounted in a xylene-based mounting gel. Slides were allowed to dry until the mounting medium had set, and then viewed under a light microscope.

The combined staining method results in a blue/ purple colour for Batrachochytrium dendrobatidis, orange for keratin and pre-keratin, and green for collagen and other sub-epidermal connective tissues.

\section{RESULTS}

Both IHC protocols clearly distinguished Batrachochytrium dendrobatidis in the skin of infected frogs 


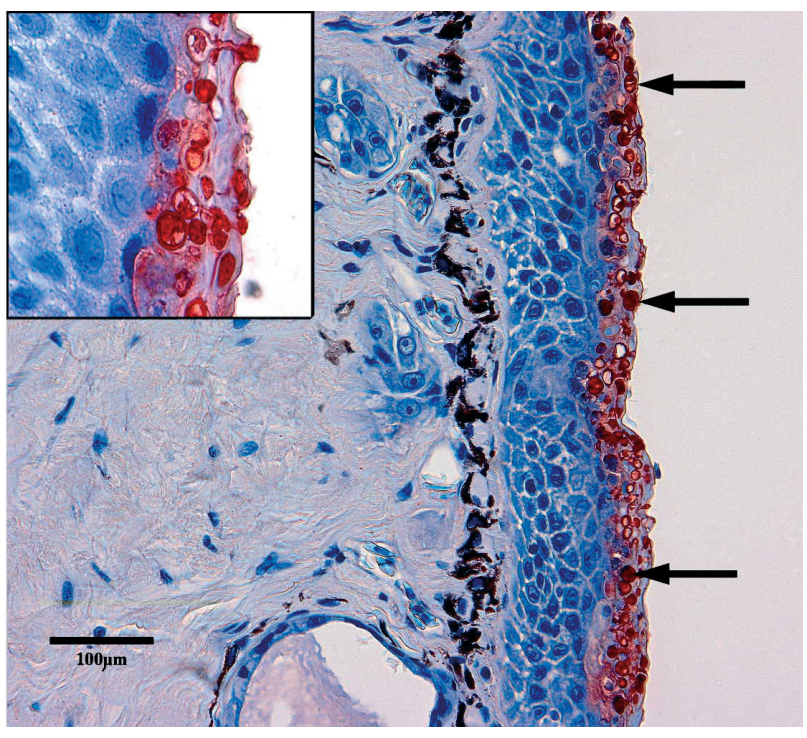

Fig. 1. Infection by Batrachochytrium dendrobatidis. Section of infected frog skin (Mixophes fasciolatus) stained using the Berger et al. (2002) IPX method. Zoosporangia (arrows) are stained red-brown with blue counterstained cells. Scale bar = $100 \mu \mathrm{m}$. Inset is an image from another sample, but at a higher magnification $(\times 3)$

(Figs. 1 \& 2). Fig. 3 illustrates the staining pattern of the modified Hollande's Trichrome stain. The complete Hollande's Trichrome staining protocol stains mitotic cells red (using $1 \%$ basic fuchsin [Fluka] diluted in $100 \%$ ethanol). As the mitotic cells are not structures that help define chytridiomycosis or hyperkeratosis,

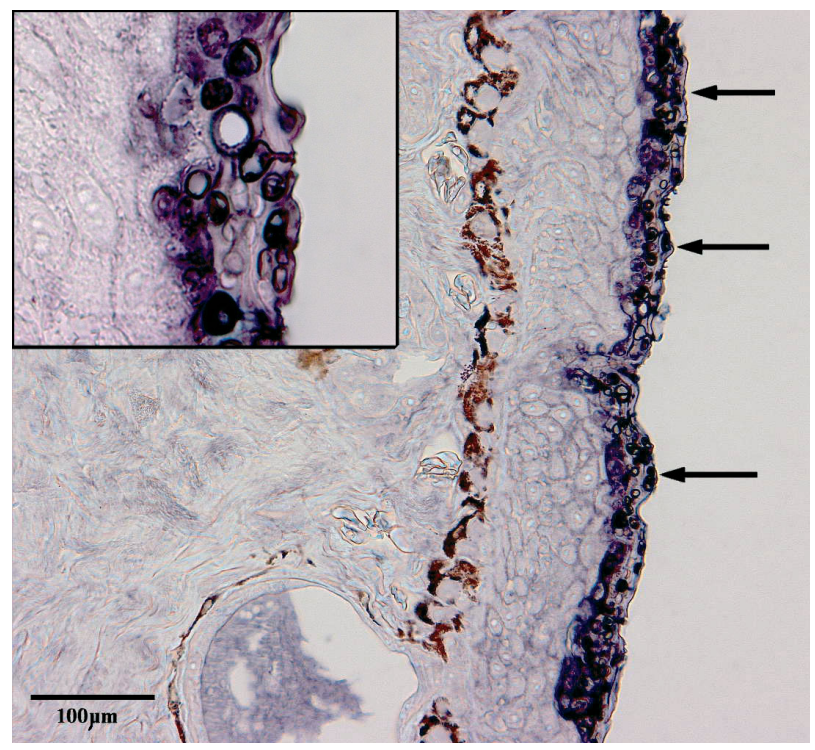

Fig. 2. Infection by Batrachochytrium dendrobatidis. Section of infected frog skin (Mixophes fasciolatus) stained using the modified Berger et al. (2002) AP method. Zoosporangia (arrows) are stained dark blue-purple. See Fig. 1 for further details

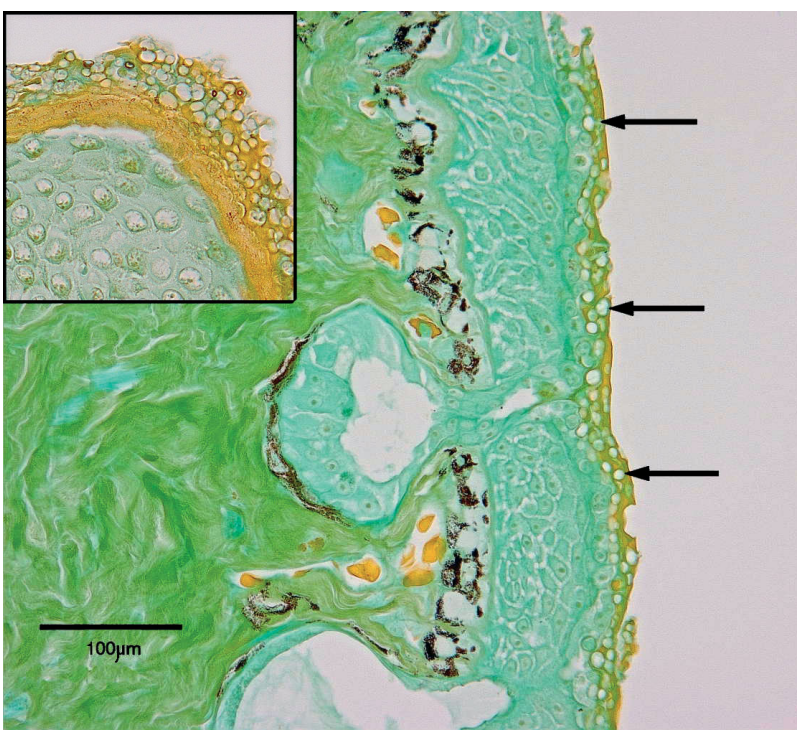

Fig. 3. Infection by Batrachochytrium dendrobatidis. Section of infected frog skin (Mixophes fasciolatus) stained with the modified Hollande's Trichrome method. Keratin is stained orange, and other sub-epidermal connective tissues are stained green. Zoosporangia (arrows) structures are not stained. See Fig. 1 for further details

and the red colour can mask other staining, the protocol was modified to stain only keratin (orange) and other sub-epidermal connective tissues (green). For combined IHC and keratin staining, the AP substrate effectively contrasts with the stained keratinised epidermis (Fig. 4).

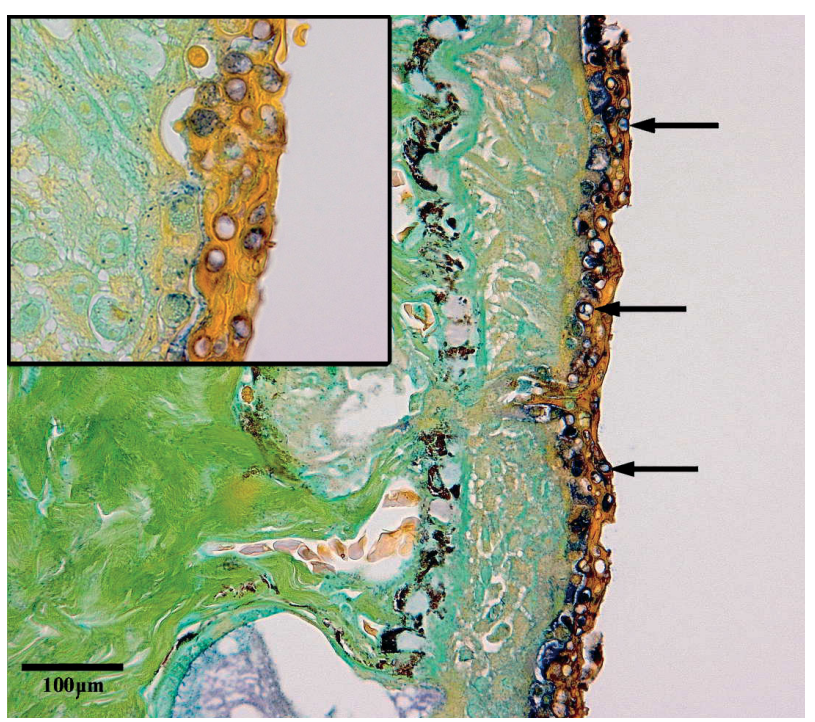

Fig. 4. Infection by Batrachochytrium dendrobatidis. Section of infected frog skin (Mixophes fasciolatus) stained with the combined IHC (AP) and modified Hollande's Trichrome keratin methods. Zoosporangia (dark blue-purple; arrows) are easily identified against the contrasting stained keratin epidermis (orange) and connective tissues (green). See Fig. 1 for further details 


\section{DISCUSSION}

AP in IHC and a modified histochemical stain (Hollande's Trichrome) demonstrates both Batrachochytrium dendrobatidis zoosporangia and keratin in frog epidermis. The advantage of this staining technique compared to previous methods is that detection of both B. dendrobatidis and keratin can be performed quickly on 1 sample. If keratin is present, the confidence in a negative diagnosis is increased, but if keratin is absent, a false negative diagnosis is possible. Modification of the Hollande's Trichrome method eliminates the need to stain slides overnight; this modification reduces the time of preparation, and also optimises the diagnosis of chytridiomycosis. Furthermore, the clear contrast in colour between $B$. dendrobatidis and the keratin enables simple diagnosis by inexperienced diagnosticians. One advantage of the new protocol is the possible reduction in time delays in diagnosis, whereby samples that may have been referred to specialist laboratories can be done locally.

Although each of these stains can be performed individually to diagnose chytridiomycosis, we suggest a more accurate diagnosis is obtained when they are used simultaneously. R. Speare (www.jcu.edu.au/ school/phtm/PHTM/frogs/chyglob.htm) found that in some animals there may be extensive sloughing of the skin, and this may result in incorrect diagnosis (false negatives) when using staining techniques such as HE since the zoosporangia are shed with the keratin. The false diagnosis may be attributable to the absence of Batrachochytrium dendrobatidis after shedding of the highly infected epidermal layer. The advantage of the new combined staining method is that it highlights the presence/absence of the keratin layer and/or B. dendrobatidis. Table 1 details how the different results generated from this technique can be interpreted.

When a negative interpretation is found, it is important to ensure that a false negative is not reported. It is recommended that more than 1 section be examined to ensure that misdiagnosis does not occur. By examining several sections, the chance of a false negative diagno-

Table 1. Possible diagnoses with the combined staining method. Ker.: keratin; B.d.: Batrachochytrium dendrobatidis

\begin{tabular}{|c|c|c|}
\hline \multicolumn{2}{|c|}{ Staining result } & \multirow[t]{2}{*}{ Interpretation } \\
\hline Ker. & B.d. & \\
\hline+ & - & $\begin{array}{l}\text { Frog was negative for } B \text {. dendrobatidis. Presence } \\
\text { of keratin allows confidence in diagnosis }\end{array}$ \\
\hline+ & + & Frog was infected with $B$. dendrobatidis \\
\hline- & - & $\begin{array}{l}\text { Equivocal. A negative identification cannot be } \\
\text { made as keratin is lacking and } B \text {. dendrobatidis } \\
\text { may be present in shed skin }\end{array}$ \\
\hline- & + & $\begin{array}{l}\text { This result has not been seen yet (frog infected } \\
\text { with } B \text {. dendrobatidis but no keratin stain) }\end{array}$ \\
\hline
\end{tabular}

Editorial responsibility: Peernel Zwart,

Utrecht, The Netherlands sis is greatly reduced. A 'negative' report refers only to the particular sections examined, i.e. the clustering of zoosporangia in the epidermis means that examination of a single or small number of sections in lightly infected frogs may result in a 'negative' result if zoospore clusters are absent in those particular sections. If the animal is deceased, then multiple samples such as toeclips and ventral skin from the limbs and inguinal areas can be sampled, thereby increasing the sampling number from the one animal.

Acknowledgements. We are very grateful to G. Russell from the AAHL histology laboratory for advice on histological stains. We thank R. Speare for reviewing a draft of this manuscript. This work was supported by funding from Integrated Research Challenges in Environmental Biology (IRCEB) grant IBN-9977063 from the National Science Foundation, USA.

\section{LITERATURE CITED}

Berger L, Speare R, Daszak P, Green DE and 10 others (1998) Chytridiomycosis causes amphibian mortality associated with population declines in the rain forests of Australia and Central America. Proc Natl Acad Sci USA 95:9031-9036

Berger L, Speare R, Hyatt AD (1999) Chytrid fungi and amphibian declines: overview, implications and future directions. In: Campbell A (ed) Declines and disappearances of Australian frogs. Environment Australia, Canberra, p 23-33

Berger L, Speare R, Kent A (2000) Diagnosis of chytridiomycosis of amphibians by histological examination. Zoos' Print $\mathrm{J}$ 15:184-190

Berger L, Hyatt AD, Olsen V, Hengstberger SG, Boyle D, Marantelli G, Humphreys K, Longcore JE (2002) Production of polyclonal antibodies to Batrachochytrium dendrobatidis and their use in an immunoperoxidase test for chytridiomycosis in amphibians. Dis Aquat Org 48:213-220

Daszak P, Berger L, Cunningham AA, Hyatt AD, Green DE, Speare R (1999) Emerging infectious diseases and amphibian population declines. Emerg Infect Dis 5:735-748

Fellers GM, Green DE, Longcore JE (2001) Oral chytridiomycosis in the mountain yellow-legged frog (Rana muscosa). Copeia 4:945-953

Gray P (1954) The microtomist's formulary and guide. Blakiston, New York

Longcore JE, Pessier AP, Nichols DK (1999) Batrachochytrium dendrobatidis gen. et sp. nov., a chytrid pathogenic to amphibians. Mycologia 91:219-227

Morehouse EA, James TY, Ganley AR, Vilgalys R, Berger L, Murphy PJ, Longcore JE (2003) Multilocus sequence typing suggests the chytrid pathogen of amphibians is a recently emerged clone. Mol Ecol 12:395-403

Nichols DK, Pessier AP, Longcore JE (1998) Cutaneous chytridiomycosis in amphibians: an emerging disease? Proc Am Assoc Zoo Veterinarians (AAZV) and Am Assoc Wildl Veterinarians (AAWV) Joint Conference, p 269-271

Nichols DK, Lamirande EW, Pessier AP, Longcore JE (2001) Experimental transmission of cutaneous chytridiomycosis in dendrobatid frogs. J Wildl Dis 37:1-11

Powell MJ (1993) Looking at mycology with a Janus face: a glimpse at Chytridiomycetes in the environment. Mycologia 85:1-20

Sparrow FK (1960) The aquatic phycomycetes. University of Michigan Press, Ann Arbor, MI

Submitted: April 15, 2004; Accepted: August 3, 2004

Proofs received from author(s): September 6, 2004 